\title{
EphB2 and ERK signaling are required for heterotypic contact inhibition of locomotion to drive cell sorting
}

Simon Brayford ${ }^{1}$, Eduardo Serna-Morales ${ }^{1}$, Andrei Luchici ${ }^{2}$, Toru Hiratsuka ${ }^{3}$ and Brian M. Stramer ${ }^{1 *}$

${ }^{1}$ Randall Centre for Cell and Molecular Biophysics, King's College London, SE1 1UL, London, United Kingdom

${ }^{2}$ Dacian Consulting, 84 Brookwood Road, SW18 5BY, London, United Kingdom

${ }^{3}$ Centre for Stem Cells and Regenerative Medicine, King's College London, SE1 9RT, London, United Kingdom

* Corresponding author / lead contact: brian.m.stramer@kcl.ac.uk

\section{Summary}

1 Interactions between different cell-types can induce distinct contact inhibition of

2 locomotion (CIL) responses that are hypothesized to control population-wide

3 behaviors during embryogenesis [1, 2]. However, our understanding of the signals

4 that lead to cell-type specific repulsion, and the precise capacity of heterotypic CIL

5 responses to drive emergent behaviors is lacking. Using a new in vitro model of

6 heterotypic CIL between epithelial and mesenchymal cells, we show that

7 fibrosarcoma cells (HT1080), but not fibroblasts (NIH3T3), are actively repelled by

8 epithelial cells in culture. We show that knocking down EphB2 in fibrosarcoma cells

9 specifically leads to disruption of the repulsion phase of $\mathrm{CIL}$ in response to

10 interactions with epithelial cells. Furthermore, this heterotypic interaction requires

11 ERK activation, downstream of EphB2 signaling. We also examine the population-

12 wide effects when these various cell combinations, and their specific heterotypic CIL

13 responses, are allowed to interact in culture. Mixtures of fibrosarcoma and epithelial

14 cells - unlike fibroblasts and epithelial cells - lead to complete sorting and segregation of the two populations, and inhibiting their distinct CIL response by knocking down EphB2 or ERK in fibrosarcoma cells disrupts this emergent sorting

17 behavior. Our understanding of the mechanisms underlying developmental 
behaviors such as cell sorting is lacking as predominant sorting hypotheses, such as differential adhesion, have recently been found inadequate in predicting the sorting of mesenchymal cells $[3,4]$. These data suggest that heterotypic CIL responses, in conjunction with processes such as differential adhesion, may aid the sorting of cell populations during embryogenesis.

\section{Results and Discussion}

Fibroblasts and fibrosarcoma cells exhibit distinct responses upon collision

\section{with an epithelial cell monolayer}

To study heterotypic cell-cell collisions, we developed a confrontation assay whereby two different cell-types are separated by a barrier, which upon removal, creates a uniform gap into which the different cell populations migrate and collide. Following a screen of a range of different epithelial versus mesenchymal cell-types, an interesting and unexpected phenomenon was revealed. When a population of migrating epithelial cells ( $\mathrm{HaCaT}$ ) encountered a population of migrating fibroblasts (NIH3T3), both populations ceased their forward migration, forming a sharp border (Figure 1A, B and Video S1). This is in stark contrast to fibrosarcoma cells (HT1080) which, upon collision with epithelial cells, seemed to undergo a complete repulsion (Figure 1A, B and Video S1). This result was not only seen with HaCaT epithelial cells, as fibrosarcoma collision with a human corneal epithelial cell line [5] also resulted in repulsion of the fibrosarcoma population (Figure S1). When no colliding partner was encountered, HaCaT epithelial cells migrated with a persistently

41 increasing speed, which was similarly observed after their collision with fibrosarcoma cells, suggesting that epithelial cell motion was unaffected by their collision with the 
43 fibrosarcoma population (Figure 1C, D). In contrast, epithelial migration was severely reduced shortly after their collision with fibroblasts (Figure 1D). The repulsion of fibrosarcoma cells was further analyzed using particle image velocimetry (PIV) to track the entire cell population, which revealed a population-wide increase in the speed of the epithelial cells following collision with fibrosarcoma cells (Figure 1E and Video S2) showing that these cells have a distinct heterotypic response to epithelial cell collisions.

Fibrosarcoma cells undergo active repulsion from epithelial cells

In order to determine whether the repulsion of fibrosarcoma cells by epithelial cells was an active process, we generated time-lapse movies of individual mesenchymal cells undergoing collisions with epithelial monolayers (Video S3) and examined their repulsive dynamics. An analysis of acceleration changes around collisions revealed that when fibrosarcoma cells collided with epithelial cells (i.e. heterotypic collisions), there was a sudden backward acceleration with respect to the colliding partner (Figure 2A) suggesting a sudden change in cell motion. This response was similar to previously reported homotypic collisions between Drosophila macrophages in vivo, which also undergo a classical CIL response involving active repulsion [6, 7]. The backward acceleration of fibrosarcoma cells was accompanied by a shift in the direction of their velocities before, during, and after the collision as 63 the fibrosarcoma cells were repelled and migrated away from the epithelial cells 64 (Figure 2B). In contrast, this repulsion was not observed when fibroblasts collided with epithelial cells, nor during fibrosarcoma-fibrosarcoma cell collisions (i.e. homotypic collisions), which led to cells continuing to migrate toward the colliding 
distance from the point of collision over time revealed that heterotypic fibrosarcoma collisions led to a slowing of their motility before migrating away from epithelial cells, in contrast to homotypic collisions, which led to their continued forward motion (Figure 2C). These data highlight that fibrosarcoma cells show distinct CIL dynamics in response to collision with epithelial cells, which involves active cell repulsion.

\section{ERK activation downstream of EphB2 induces heterotypic repulsion of fibrosarcoma cells}

There is much evidence in the literature for Eph receptors playing a role in epithelial cell interactions. EphA2 for example has been shown to drive the segregation of Ras-transformed epithelial cells from normal neighbors [8] and HEK293 cells overexpressing EphB2 have been shown to segregate from the same cells overexpressing ephrin-B1 [9]. However, less is known about whether ephrin signaling may control the repulsion and segregation of mesenchymal cell populations, similar to epithelial monolayers. Indeed, HT1080 fibrosarcoma cells express the Eph receptor EphB2, knockdown of which in these cells (Figure S2A) abolished the backward acceleration upon their collision with epithelial cells (Figure 2A). Furthermore, knockdown of EphB2 in fibrosarcoma cells led to a random distribution of the direction of velocities after collision suggesting that cells were randomly migrating away from epithelial cells rather than being actively repelled. (Figure 2B). Similarly, plotting nuclear distance from the point of collision over time revealed that EphB2 knockdown in fibrosarcoma cells slowed their separation from epithelial cells further showing that the repulsion phase of CIL was disrupted (Figure 2D). We also tested the effect of knocking down EphB2 in the confrontation assay and found that indeed, collective repulsion of fibrosarcoma cells following collision 
with a monolayer of epithelial cells was also disrupted (Figures 2E and S2B).

One signaling pathway previously reported to be controlled by Eph receptor activation is the ERK pathway. However, there is conflicting data for the role of ERK signaling specifically downstream of Eph receptor activation, with some reports suggesting an inhibition of ERK activity $[10,11]$ and others reporting an increase in ERK activity following Eph receptor stimulation [12-14]. We found that knockdown of ERK1/2 in fibrosarcoma cells (Figure S2C) phenocopied the results of EphB2 knockdown both in single-cell collisions (Figure 2A, B and D) and in confrontation assays (Figures 2E and S2B), showing that a combination of EphB2 activation and ERK signaling is required for fibrosarcoma repulsion from epithelial cells. In order to confirm that the effects of knocking down EphB2 or ERK signaling were indeed related to cell collisions and not general migration defects, we measured the speed and persistence of freely moving, non-colliding fibrosarcoma cells and found no significant difference in either speed (Figure S2D) or persistence (Figure S2E) in EphB2 or ERK knockdown cells suggesting that knockdown only affected events related to contact with another cell, rather than general migration. Interestingly, myosin II, which has been reported to be downstream of ERK signaling [15-17] and involved in Eph-mediated repulsion of other cell-types [18] did not appear to play a role in fibrosarcoma repulsion from epithelial cells as myosin inhibition with Blebbistatin had no effect in the confrontation assay (Figure S3).

We next examined ERK activity during heterotypic collisions using a mixing assay whereby two cell-types were combined 1:1, seeded together, and allowed to interact over time. Lysates from mixed-cell populations were then collected after 24 $\mathrm{h}$ and examined for ERK activity. Western blot analysis of these lysates revealed that phospho-ERK (pERK) was highest among epithelial-fibrosarcoma co-cultures, 
118 which was increased compared with either of these cell types individually (Figure

$1193 \mathrm{~A})$ suggesting that one population was contributing to ERK activation in the other.

120 We also found that EphB2 knockdown in fibrosarcoma cells, whilst not affecting total

121 ERK levels (Figure 3B), resulted in a significant decrease in levels of pERK in

122 epithelial-fibrosarcoma co-cultures (Figure $3 \mathrm{C}$ ), suggesting that ERK activation is

123 downstream of EphB2 in the fibrosarcoma population.

$124 \quad$ While western blotting shows pERK levels in an entire cell population, it provides no information as to ERK activation dynamics during individual cell-cell interactions. For this we used a FRET biosensor which has previously been

127 demonstrated to report ERK activity in living cells [19]. In addition, this technique 128 allows measurement of ERK activity in specific subpopulations of cells where western blotting does not. Following delivery of the biosensor into the fibrosarcoma population we repeated the confrontation assay and found that in fibrosarcoma cells

131 at the migrating front, ERK activity was significantly increased after collision with 132 epithelial cells (Figure 3D). We next analyzed time-lapse movies of the mixing assay 133 and found an overall higher level of active ERK among fibrosarcoma cells in the 134 presence of epithelial cells compared to fibrosarcoma cells alone (Figure 3E).

135 Finally, we found that cells in the mixed group had a higher fluctuation in their ERK 136 activity over time (by measuring variance in ERK activity of cell tracks) compared to 137 fibrosarcoma only (Figure 3F). This was likely due to fibrosarcoma cells constantly 138 undergoing heterotypic collisions with epithelial cells throughout the course of the assay. Taken together, these data suggest that ERK signaling acts downstream of

140 EphB2 and is involved in the repulsion of fibrosarcoma cells upon their contact with 141 epithelial cells. 
143 Fibrosarcoma and epithelial cell populations sort in culture through CIL

\section{4 interactions}

145 To examine how distinct heterotypic and homotypic CIL dynamics affects the

146 behavior of a mixed population of cells, we repeated the mixing assay and examined

147 the population dynamics by generating time-lapse movies. We found that epithelial 148 cells and fibroblasts mixed to form a homogenous, interspersed population, in 149 contrast to epithelial and fibrosarcoma cells, which immediately began to sort from 150 one another (Figure 4A and Video S4). In addition, automated tracking of the 151 mesenchymal cells in these movies revealed a streaming-type behavior specifically 152 among fibrosarcoma cells (Figure 4B and Video S4), highlighting this population's 153 segregation from epithelial cells over time. To examine whether the sorting behavior of fibrosarcoma cells was due in part to their collective migration, we examined the correlation in the direction of local instantaneous cell velocities, which measures the coupling of motion among neighboring cells in the population. This revealed that,

157 while the local cell velocities of fibrosarcoma cells were more highly correlated than

158 fibroblasts, fibrosarcoma cells still did not display a strong correlation in their motion

159 (fibrosarcoma velocity correlation $=0.16 \pm 0.01$; fibroblast velocity correlation $=0.08$

$160 \pm 0.01$; mean \pm SEM; 1 = perfect correlation, $-1=$ perfect anti-correlation) (Figure S4A and B). This suggests that, rather than physically following one another, fibrosarcoma cells are likely segregating from epithelial cells through some other mechanism.

In order to examine whether the specific repulsive CIL dynamics of

165 fibrosarcoma cells in response to epithelial cells was driving their segregation we 166 inhibited Eph and ERK signaling in the fibrosarcoma population. Indeed, knockdown 167 of EphB2 or ERK1/2 in fibrosarcoma cells resulted in a disruption to their 
segregation from epithelial cells as quantified by measuring the overall dispersion of epithelial cells (Figure 4C and D). Similarly, treatment with the MEK inhibitor, U0126, also resulted in disruption to the segregation between fibrosarcoma and epithelial cells (Figure S4C and E) further supporting a role for ERK signaling in the repulsion and segregation of these two populations. These data suggest that segregation of fibrosarcoma from epithelial cells is driven by the repulsive interaction between these two cell populations.

Finally, we investigated the phenomenon of cell-sorting over a longer timecourse to examine the final patterns of the cell populations. When epithelial cells were plated sparsely and grown over a four-day period, they grew in clusters that eventually merged to form large, sheet-like structures (Figure 4E). However, epithelial cells grown in the presence of fibroblasts resulted in much smaller clusters with irregular shapes, as the two cell populations reached equilibrium (Figure 4E). In contrast, when grown together with fibrosarcoma cells, epithelial cells formed large colonies, seemingly unimpeded by the presence of fibrosarcoma cells (Figure 4E). Indeed, quantification of epithelial cell area over time revealed that their proliferation was specifically reduced in the presence of fibroblasts suggesting that epithelial cells may undergo contact inhibition of proliferation in the absence of a repulsive heterotypic CIL response (Figure 4F). We found no appreciable difference in basal proliferation rate between fibroblasts and fibrosarcoma cells (Figure S4F), suggesting that differential CIL dynamics was allowing fibroblasts, but not fibrosarcoma cells to out-compete epithelial cells for space, rather than a difference in their respective proliferation rates. Importantly, the ability of fibrosarcoma cells to almost completely segregate from epithelial cells differs from other 2D models of epithelial sorting in which epithelial cells do not undergo complete segregation, but 
193 rather lead to an intermediate aspect of separation that locks in small clusters of 194 cells $[9,20,21]$.

The differential adhesion hypothesis (DAH) has been the predominant model

196 to explain the sorting of cell populations during development. Two different cell types

197 can theoretically segregate simply by taking into account their differential adhesion

198 (and differential surface tension) [22]. This model assumes that the cells in question segregate in a liquid-like phase separation guided by the reduction in adhesive-free energy as the two cell-types maximize their cell-cell adhesions. The problem with this concept in isolation is that we now know that groups of cells do not behave like perfect liquids; clusters of cells can quickly go from a fluid-like to a solid-like state as cell density increases in a process termed 'jamming', which leads to cells rapidly slowing their motion [23]. Therefore, DAH can only work in a regime where the cells are not proliferating and at a sweet spot in their density that allows sufficient fluidity to the tissue. Otherwise the cells will not be allowed to test the adhesive landscape sufficiently enough to lead to complete segregation. This is likely to be particularly important for epithelial populations (the predominant cell-type experimentally tested during cell sorting), which are not highly motile due to their strong cell-cell adhesions.

211 completely segregate from an epithelial population in 2D culture. Differential

212 adhesion in this case is still playing a role in the separation due to the high surface

213 tension of the epithelial population and the nearly non-existent mutual adhesion of

214 the fibrosarcoma cells leading to the less cohesive cell (HT1080) surrounding the 215 more cohesive cell type (HaCaT) as expected by the DAH [22]. However, DAH is not

216 sufficient on its own; inhibiting the repulsion between these two cell types prevents 217 their sorting behavior. We hypothesize that the strong heterotypic CIL response 
218 between fibrosarcoma and epithelial cells essentially helps fluidize the population

219 allowing the cells to sample their adhesive surroundings. Furthermore, there also

220 appears to be an aspect of fibrosarcoma CIL relieving contact inhibition of

221 proliferation of the epithelial cells (possibly through a reduction in spatial constraints

222 on the epithelial clusters) allowing epithelial colonies to grow and merge, which

223 would also enhance the fluidity of the population. It is interesting to note that the

224 other mesenchymal cell-type (NIH3T3) failed to segregate from the epithelial

225 population as one would expect for a mixture of highly cohesive and non-cohesive

226 cells [22]. In the fibroblast-epithelial combination, the epithelial cells are highly

227 inhibited in their proliferation (likely due to a lack of heterotypic repulsion), which

228 would enhance the spatial constraint on the epithelial colonies. In this case the lack

229 of a repulsive CIL response prevents the epithelial colonies from growing enough to

230 merge with neighboring colonies and thus separate from the fibroblast population. It

231 is therefore possible that we are also highlighting an unexplored connection between

232 contact inhibition of locomotion and contact inhibition of proliferation in this model

233 despite the fact that these two processes are largely thought to be distinct behaviors

$234[1]$.

235 CIL in recent years has been shown to play a role in the migration and

236 patterning of numerous cell types during development. These data suggest that a

237 repulsive CIL response in conjunction with other behaviors, such as differential

238 adhesion, may also enhance the sorting of cell populations during embryogenesis.

240 Acknowledgements

241 We thank Vicky Sanz-Moreno, Matthias Krause and Stephen Terry for use of cell-

242 lines as well as Fiona Watt and Michiyuki Matsuda for use of and assistance with the 
243 ERK FRET biosensor. We would also like to thank Claudia Linker for feedback in

244 writing the manuscript. This project has received funding from the European

245 Research Council (ERC) under the European Union's Horizon 2020 research and

246 innovation program grant agreement No. 681808. TH has received funding from the

247 European Union's Horizon 2020 research and innovation program under the Marie

248 Sklodowska-Curie grant agreement No. 704587.

\section{Author Contributions}

Conceptualization, S.B. and B.M.S.; Methodology, S.B.; Software, E.S-M. and A.L.;

Formal Analysis, S.B., E.S-M. and T.H.; Investigation, S.B.; Writing - Original Draft,

S.B.; Writing - Review \& Editing, S.B. and B.M.S.; Project Administration, B.M.S.;

Funding Acquisition, B.M.S.

\section{Declaration of Interests}

257 The authors declare no competing interests.

\section{References}

260 1. Stramer, B., and Mayor, R. (2016). Mechanisms and in vivo functions of contact inhibition of locomotion. Nature reviews. Molecular cell biology.

2. Theveneau, E., Steventon, B., Scarpa, E., Garcia, S., Trepat, X., Streit, A., and Mayor, R. (2013). Chase-and-run between adjacent cell populations promotes directional collective migration. Nat Cell Biol 15, 763-772.

3. Pawlizak, S., Fritsch, A.W., Grosser, S., Ahrens, D., Thalheim, T., Riedel, S.,

4. Tambe, D.T., and Fredberg, J.J. (2015). And I hope you like jamming too. 
5. Terry, S.J., Dona, F., Osenberg, P., Carlton, J.G., and Eggert, U.S. (2018). Capping protein regulates actin dynamics during cytokinetic midbody maturation. Proc Natl Acad Sci U S A 115, 2138-2143.

6. Davis, J.R., Luchici, A., Mosis, F., Thackery, J., Salazar, J.A., Mao, Y., Dunn, G.A., Betz, T., Miodownik, M., and Stramer, B.M. (2015). Inter-cellular forces orchestrate contact inhibition of locomotion. Cell 161, 361-373.

7. Davis, J.R., Huang, C.Y., Zanet, J., Harrison, S., Rosten, E., Cox, S., Soong, D.Y., Dunn, G.A., and Stramer, B.M. (2012). Emergence of embryonic pattern through contact inhibition of locomotion. Development 139, 4555-4560.

9. Poliakov, A., Cotrina, M.L., Pasini, A., and Wilkinson, D.G. (2008). Regulation of EphB2 activation and cell repulsion by feedback control of the MAPK pathway. J Cell Biol 183, 933-947.

10. Miao, H., Nickel, C.H., Cantley, L.G., Bruggeman, L.A., Bennardo, L.N., and Wang, B. (2003). EphA kinase activation regulates HGF-induced epithelial branching morphogenesis. J Cell Biol 162, 1281-1292.

11. Elowe, S., Holland, S.J., Kulkarni, S., and Pawson, T. (2001). Downregulation of the Ras-mitogen-activated protein kinase pathway by the EphB2 receptor

12. Pratt, R.L., and Kinch, M.S. (2002). Activation of the EphA2 tyrosine kinase stimulates the MAP/ERK kinase signaling cascade. Oncogene 21, 7690-7699. tyrosine kinase is required for ephrin-induced neurite retraction. Mol Cell Biol

13. Vindis, C., Cerretti, D.P., Daniel, T.O., and Huynh-Do, U. (2003). EphB1 recruits $\mathrm{c}$-Src and p52Shc to activate MAPK/ERK and promote chemotaxis. J Cell Biol 162, 661-671.

14. Kandouz, M., Haidara, K., Zhao, J., Brisson, M.L., and Batist, G. (2010). The 
304

16. Kamioka, Y., Sumiyama, K., Mizuno, R., Sakai, Y., Hirata, E., Kiyokawa, E., and Matsuda, M. (2012). Live imaging of protein kinase activities in transgenic mice expressing FRET biosensors. Cell Struct Funct 37, 65-73.

17. Mizuno, R., Kamioka, Y., Kabashima, K., Imajo, M., Sumiyama, K., Nakasho, E., Ito, T., Hamazaki, Y., Okuchi, Y., Sakai, Y., et al. (2014). In vivo imaging reveals PKA regulation of ERK activity during neutrophil recruitment to inflamed intestines. J Exp Med 211, 1123-1136.

18. Fagotto, F., Rohani, N., Touret, A.S., and Li, R. (2013). A molecular base for cell sorting at embryonic boundaries: contact inhibition of cadherin adhesion by ephrin/ Eph-dependent contractility. Dev Cell 27, 72-87.

19. Komatsu, N., Aoki, K., Yamada, M., Yukinaga, H., Fujita, Y., Kamioka, Y., and Matsuda, M. (2011). Development of an optimized backbone of FRET biosensors for kinases and GTPases. Mol Biol Cell 22, 4647-4656.

20. Taylor, H.B., Khuong, A., Wu, Z., Xu, Q., Morley, R., Gregory, L., Poliakov, A., Taylor, W.R., and Wilkinson, D.G. (2017). Cell segregation and border sharpening by Eph receptor-ephrin-mediated heterotypic repulsion. J R Soc Interface 14.

21. Taylor, W.R., Morley, R., Krasavin, A., Gregory, L., Wilkinson, D.G., and Poliakov, A. (2012). A mechanical model of cell segregation driven by differential adhesion. PLoS One 7, e43226.

22. Foty, R.A., and Steinberg, M.S. (2013). Differential adhesion in model systems. Wiley Interdiscip Rev Dev Biol 2, 631-645.

23. Sadati, M., Taheri Qazvini, N., Krishnan, R., Park, C.Y., and Fredberg, J.J. (2013). Collective migration and cell jamming. Differentiation 86, 121-125.

24. Betz, T., Koch, D., Lim, D., and Kas, J.A. (2009). Stochastic actin polymerization and steady retrograde flow determine growth cone advancement. Biophys J 96, 5130-5138.

25. Dunn, G.A., and Paddock, S.W. (1982). Analysing the motile behaviour of cells: a general approach with special reference to pairs of cells in collision. Philos Trans R Soc Lond B Biol Sci 299, 147-157. 


\section{Materials \& Methods}

339

\section{Cell culture}

341 Immortalized human keratinocytes (HaCaT), immortalized mouse fibroblasts

342 (NIH3T3), human fibrosarcoma (HT1080) and immortalized human corneal epithelial

343 cells [5] were all maintained in Dulbecco's Modified Eagle's Medium (DMEM) with

$3444500 \mathrm{mg} / \mathrm{L}$ glucose, supplemented with $10 \% \mathrm{FBS}$, at $37^{\circ} \mathrm{C}$ and $5 \% \mathrm{CO}_{2}$. For routine maintenance, cells were cultured in T75 plastic cell culture flasks and split via trypsinization approximately every 3 days or when approaching confluence.

\section{Cell labelling}

348 CellTracker Green CMFDA and CellTracker Red CMTPX dyes (Invitrogen, Carlsbad,

349 CA) were used to differentially label cell-types in mixed-cell assays. Cells were exposed to either dye at $1 \mu \mathrm{M}$ in serum-free medium for 30 mins at $37^{\circ} \mathrm{C}$ before being washed once with PBS, trypsinized, re-suspended in complete medium and

352 counted for downstream purpose.

\section{$353 \quad$ Live imaging}

354 Movies were acquired using an LSM 880 inverted confocal microscope (Zeiss,

355 Oberkochen, Germany). Cells were maintained at $37^{\circ} \mathrm{C}$ and $5 \% \mathrm{CO}_{2}$ for the 356 duration of live imaging. Images were acquired using differential interference 357 contrast (DIC) imaging along with airyscan filter sets for 488 or 561 lasers with either 358 a $40 X$ (NA 0.95) oil or 20X (NA 0.45) air objective (Zeiss).

\section{Confrontation assay}

$360 \quad 2$-well culture inserts (Ibidi, Germany) were placed into the centre of the well of a 24

361 well $\mu$-Plate (Ibidi, Germany). Two different pre-labelled cell-types were seeded into

362 opposite chambers at a density of $1 \times 10^{5}$ cells $/ \mathrm{cm}^{2}$ and incubated overnight. The 
363 culture insert was then carefully removed and the well topped up with $1 \mathrm{~mL}$ complete

364 culture medium before live imaging of the resulting $500 \mu \mathrm{m}$ gap.

\section{Particle image velocimetry (PIV)}

366 Time-lapse images of HaCaT and HT1080 cells from the confrontation assay movie

367 (presented in Figure 1A) were manually segmented prior to analysis and pseudo368 speckle analysis was performed as described previously [24]. For pseudo-speckle analysis, the size of the search image was chosen such that it spanned the maximum expected displacement of the cells during the acquisition time. To cover

371 the whole search image, a cross-correlation coefficient was computed between

372 source image and a sub-image of the search image shifted by one pixel. To remove

373 anomalous tracking data, only displacements that had a cross-correlation coefficient above a certain threshold were kept. Finally, a spatial convolution with a Gaussian kernel and temporal convolution were used to interpolate the measured displacements to cover all the pixels within the frame. The complete algorithm for

377 this analysis, including the filtering and interpolation was implemented in MatLAB $378 \quad\left(\right.$ MathWorks $\left.{ }^{\circledR}\right)$.

\section{Individual cell collisions and manual tracking}

380 Analysis of single-cell collisions was carried out by mixing pre-labelled cells at low

381 density, plating sparsely and allowing to adhere $4 \mathrm{~h}$ before imaging every $1 \mathrm{~min}$ at 382 20X. For the kinematics analysis, a collision was defined as the moment the the cell 383 in question contacts any part of the colliding partner. The centroid of the cell nucleus, which could be easily identified from DIC movies was manually tracked using the mTrackJ plugin for ImageJ $(\mathrm{NIH})$ to calculate the $x, y$ coordinates of the cell

386 at all time-points.

\section{$387 \quad$ Kinematics analysis}


388 Kinematics analysis of the velocity and acceleration of cells was calculated as

389 previously described [6, 25]. In order to assess the statistical significance of the

390 direction of cells after collision, a binomial test with a probability of success of $95 \%$

391 was performed on the cell velocity unit vectors every five minutes from 5 min before

392 to 20 min after collision. To assess the statistical significance of acceleration, a one-

393 sample $t$ test of the horizontal component of the vectors was performed. This test

394 analyzes how far from zero the displacement average of the horizontal component

395 of the vectors is at the collision time. If the average is similar to zero it suggests that

396 the displacements are random.

\section{Gene silencing by small interfering RNA (siRNA)}

398 HT1080 cells were plated onto 6 -well plates at $2 \times 10^{5}$ cells / well and allowed to 399 attach overnight. Cells were transfected with pre-validated siRNA sequences to 400 knockdown human EphB2 (Sigma, cat. no. EHU060511) or human p44/42 MAPK 401 (ERK1/2) (Cell Signaling Technologies, Cat no. 6560). Mock transfection was used 402 as a negative control. siRNA was transfected using Oligofectamine $\AA$ reagent 403 (Invitrogen) according to the manufacturer's instructions. Experiments were carried out $48 \mathrm{~h}$ post-transfection.

\section{Western blotting and antibodies}

406 Total cellular proteins from individual cells or mixed-cell populations were prepared

407 by rinsing cells with cold PBS and scraping with RIPA buffer (20 mM Tris pH 7.4, $408150 \mathrm{mM}$ sodium chloride, $1 \%$ (v/v) Nonidet P-40, 0.5\% (w/v) sodium deoxycholate, 1 $409 \mathrm{mM}$ EDTA, $0.1 \%(\mathrm{w} / \mathrm{v}) \mathrm{SDS})$ in the presence of protease and phosphatase inhibitor 410 cocktails (Roche Diagnostics, Indianapolis, IN). Approximately equal amounts of 411 protein $(20 \mu \mathrm{g})$ were resolved on $12.5 \%$ SDS-PAGE gels before electro-transfer to 412 PVDF membranes. Following blocking in 5\% (w/v) BSA in TBST, immunoblotting 
413 was performed using anti-EphB2 (CST, \#83029), anti-GADPH (Millipore, \#ABS16),

414 anti-p44/42 MAPK (CST \#9102), anti-cofilin (CST \#5175) or anti-Phospho-p44/42

415 MAPK to detect EphB2, GAPDH, ERK1/2, cofilin and pERK1/2 (Thr202/Tyr204)

416 protein respectively. The membranes were then washed with TBST and incubated

417 with species appropriate HRP-conjugated IgG secondary antibodies (Agilent

418 Technologies, Santa Clara, CA). Chemiluminescence was measured using ImageJ

419 (NIH) after applying Clarity ${ }^{\text {TM }}$ Western ECL substrate (BioRad, Hercules, CA).

\section{ERK FRET biosensor plasmid}

421 The lentiviral plasmid of nucleus-localized FRET biosensor for ERK (EKAREV-NLS)

422 has been previously characterized [19] and was a gift from Dr. Michiyuki Matsuda at 423 Kyoto University, Japan.

424 Lentiviral transduction

425 EKAREV-NLS ERK biosensor was expressed in HT1080 cells by lentiviral 426 transduction. EKAREV-ELS in replication-defective, self-inactivating lentiviral pCSII 427 vector was co-transfected with packaging plasmid (pCAG-HIVgp) and VSV-G-/Rev428 expressing plasmid (pCMV-VSVG-RSV-Rev) into Lenti-X 293T cells (Clontech). 429 High-titre viral solution was prepared and used for transduction into HT1080 cells.

\section{$430 \quad$ Cell mixing assay}

431 Cells were pre-labelled with either cell-tracker red or green, counted and mixed in

$4321: 1$ suspensions so that $5 \times 10^{4}$ of each cell type was seeded per well in a 24-well 433 imaging $\mu$-Plate (total of $1 \times 10^{5}$ cells / well). Cells were allowed $6 \mathrm{~h}$ to adhere before 434 being imaged overnight for a total of $22 \mathrm{~h}$ (for the wild-type analysis). For the RNAi 435 mixing assay, cells were allowed to mix for $24 \mathrm{~h}$ before being fixed with $4 \%$ 436 paraformaldehyde. All nuclei were then labelled with DRAQ5 (Invitrogen) before 437 coverslips were mounted. Still images were then acquired and the green channel 
was used to create a mask over the HT1080 cells' nuclei, leaving only the HaCaT cells' nuclei visible and then using the nearest neighbour distance calculation in

$440 \quad$ ImageJ $(\mathrm{NIH})$.

\section{Automated cell tracking and alignment analysis}

442 For automated tracking, the image channel corresponding to NIH3T3 or HT1080 443 cells was thresholded in ImageJ so that individual cells could be detected as 444 particles in the TrackMate plugin and tracks generated. The nearest neighbor analysis obtains the correlation between the instant displacement vectors of pairs of nearest neighbor cells at every frame. First, pairs of nearest neighbors are found,

447 afterwards, each cell is tracked onto the next frame to generate an instant 448 displacement vector. Posteriorly, a Pearson correlation is performed to find the linear correlation between the instant displacement vector of each pair of neighbors, which can be represented as the cosine of the angle formed by two vectors. To represent the correlation data, an average of the correlation coefficient of all pairs of nearest neighbors at each frame was performed.

\section{Long-term co-culture assay}

HaCaT cells were pelleted by centrifugation and re-suspended in CellTrace ${ }^{\text {TM }}$ CFSE (Invitrogen) at a working concentration of $5 \mu \mathrm{M}$ in PBS and incubated for 20 minutes at $37^{\circ} \mathrm{C}$. Cells were again pelleted and re-suspended in fresh culture medium to a

457 density of $2 \times 10^{4}$ cells / $\mathrm{mL}$ and incubated for 10 minutes to allow the CFSE reagent 458 to undergo acetate hydrolysis. This suspension was then mixed with either DMEM 459 (control), an NIH3T3 cell suspension (unlabelled) or HT1080 cell suspension 460 (unlabelled) and added to a 24-well plate for a total of 20,000 cells per well and 461 incubated for 4 days, fixing with $4 \%$ paraformaldehyde every $24 \mathrm{~h}$ and mounting 462 coverslips with ProlongGold (Invitrogen). Coverslips were then imaged with a 20x 
463 objective for analysis of HaCaT growth area using ImageJ (NIH).

\section{Proliferation assay}

465 NIH3T3 and HT1080 cells were seeded in a 96-well plate at 1000 cells per well and

466 incubated for 4 h. Growth medium was then carefully removed and 1X CyQUANT $^{\circledR}$

467 NF (Invitrogen) dye reagent was added. Cells were then incubated at $37^{\circ} \mathrm{C}$ for $1 \mathrm{~h}$.

468 This incubation period is required for equilibration of dye-DNA binding, resulting in a 469 stable fluorescence endpoint. The fluorescence intensity of each sample was then

470 imaged every $24 \mathrm{~h}$ using a fluorescence microplate reader with excitation at $\sim 485$

$471 \mathrm{~nm}$ and emission detection at $\sim 530 \mathrm{~nm}$. Fresh growth medium was added every 48

472 h. Cell numbers at each time-point were determined using a standard curve as per

473 the manufacturer's instructions.

\section{Drug Treatments}

475 For the myosin II inhibition experiment, the photo-stable derivative of blebbistatin, 476 (S)-nitro-Blebbistatin (Cayman Chemical, Ann Arbor, MI) was used at a final 477 concentration of $50 \mu \mathrm{M}$ in complete medium. For MEK inhibition experiments, U0126 478 (Merck, Kenilworth, NJ) was used at a final concentration of $20 \mu \mathrm{M}$ in complete 479 medium.

\section{$480 \quad$ Statistical analyses}

481 Statistical analyses are described in each figure legend.

482

483

484

485

486

487 


\section{Figure Legends}

489

Figure 1. Fibroblasts and fibrosarcoma cells exhibit distinct responses upon collision with an epithelial cell monolayer

(A) Screenshots from representative movies of a confrontation assay in which epithelial cells (HaCaT) are allowed to collide with either fibroblasts (NIH3T3) or fibrosarcoma cells (HT1080). Epithelial cells (HaCaT) are labelled green and either fibroblasts (NIH3T3) or fibrosarcoma (HT1080) cells are labelled magenta. The red line indicates the position of initial cell-cell contact. Scale bars $=100 \mu \mathrm{m}$.

(B) Quantification of HaCaT displacement $24 \mathrm{~h}$ after colliding with NIH3T3 or HT1080 cells in the confrontation assay $\left(n=3\right.$, error bars $=$ SEM, ${ }^{* *} P<0.001$, Student's t-test).

(C) Quantification of the speed of the front row of HaCaT cells throughout the confrontation assay when no other cell population is encountered, showing a persistent increase over $24 \mathrm{~h}(\mathrm{n}=3$, error bars $=$ SEM).

(D) Quantification of the speed of the front row of HaCaT cells throughout the confrontation assay involving collision with either NIH3T3 or HT1080 cell

(E) Particle Image Velocimetry (PIV) analysis of the HaCaT and HT1080 interaction in 'A' showing an increase in speed of the entire population of HaCaT cells despite having contacted HT1080 cells. Blue to red represents a shift from low to high speed. 
Figure 2. Fibrosarcoma cells undergo active repulsion upon collision with epithelial cells which is perturbed by EphB2 or ERK1/2 knockdown

(A) Vectors depicting the acceleration changes upon collision (time $=0 \mathrm{~min}$ ) analysed using 10-minute time steps and normalized to the position of the colliding partner (denoted by the large arrow). A significant rearward acceleration is only observed in the fibrosarcoma (HT1080) vs epithelial (HaCaT) collision. (n $\geq 8$ collisions per condition. ${ }^{* *} \mathrm{P}<0.01$, non-parametric Wilcoxon signed rank test).

(B) Vectors depicting the velocity of colliding cells at 10 mins before collision, at time of collision ( $0 \mathrm{~min}$ ), and at 10 mins after collision, analysed using 5-minute time steps and normalized to the position of the colliding partner (denoted by the large arrow). A significant post-collision rearward velocity away from the colliding partner is only observed in the HT1080 vs HaCaT response. ( $n \geq 8$ collisions per condition. ${ }^{*} \mathrm{P}<0.05$, non-parametric Wilcoxon signed rank test).

(C) Quantification of the distance of the nucleus from the position at which cell-cell contact occurs during heterotypic and homotypic interactions between HT1080, NIH3T3, and HaCaT cells. Note that after collision only heterotypic interaction between HT1080 and HaCaT cells leads to a rapid increase in cell separation (n $\geq 8$ collisions per condition, error bars = SEM).

(D) Quantification of the distance of the nucleus from the position at which cell-cell contact occurs during heterotypic HaCaT/HT1080 collisions in EphB2 or ERK1/2 knockdown in HT1080 cells. Note that a reduction in EphB2 or ERK1/2 reduces cell separation following collision ( $\mathrm{n} \geq 6$ collisions per condition, error bars $=$ SEM). 
(E) Quantification of the displacement of the front row of HaCaT cells after collision with HT1080 cells in confrontation assays comparing control, and EphB2 or ERK1/2 HT1080 knockdown. Note that the reduction in EphB2 or ERK1/2 in HT1080 cells slows the migration of the HaCaT population. $(n=3$, error bars $=$

Figure 3. ERK activation is elevated in collisions between epithelial and fibrosarcoma cells

(A) Western blot for pERK in cell lysates collected at $24 \mathrm{~h}$ after plating cells individually or mixing the different cell types at a 1:1 concentration. The right panel shows a quantification of pERK levels revealing ERK activity is highest during co-culture of epithelial (HaCaT) and fibrosarcoma (HT1080) cells. $(n=3$, error bars $=$ SEM)

(B) Western blot for total ERK in cell lysates in HaCaT/HT1080 co-cultures comparing controls and EphB2 knockdown in the HT1080 population. The right panel shows a quantification of total ERK levels revealing total ERK is unaffected by EphB2 knockdown in HT1080 cells. $(n=3$, error bars $=$ SEM, ns $=$ not

(C) Western blot for pERK in cell lysates in HaCaT/HT1080 co-cultures comparing controls and EphB2 knockdown in the HT1080 population. The right panel shows a quantification of pERK revealing ERK activity is reduced in the co-culture after

(D) Quantification of ERK activity using the ERK FRET biosensor in the leading row of HT1080 cells in the confrontation assay 30 mins before collision vs 30 mins 
after collision. ( $n=28$ cells from 4 separate movies, boxplots represent range, median and quartiles, ${ }^{\star} \mathrm{P}<0.05$, Mann-Whitney test).

(E) Quantification of ERK activity from four representative tracks of HT1080 cells cultured alone (blue) or during co-culture with HaCaT cells (red). Note the increase in FRET activity in the co-cultured HT1080 population along with the increased fluctuation.

(F) Quantification of the variance in ERK activity in randomly selected tracks of HT1080 cells cultured alone or during co-culture with HaCaT cells. ( $n=15$

Figure 4. Fibrosarcoma cells segregate from epithelial cells in culture, a behavior which is disrupted by EphB2 or ERK knockdown

(A) Screenshots from movies of co-cultures of epithelial cells (HaCaT) with either tracks, line $=$ mean, error bars $\pm S D,{ }^{* \star *} \mathrm{P}<0.001$, Mann-Whitney test) fibroblasts (NIH3T3) or fibrosarcoma cells (HT1080). HaCaT cells are represented in green and mesenchymal cells in magenta. Scale bar $=100 \mu \mathrm{m}$.

(B) Tracks of NIH3T3 or HT1080 cells throughout the movies in 'A' and Video S4.

(C) Images of co-cultures (fixed at $24 \mathrm{~h}$ ) of HaCaT cells (green) with either control, EphB2 or ERK1/2 knockdown HT1080 cells (magenta). Scale Bar = $100 \mu \mathrm{m}$.

(D) The dispersion of HaCaT cells in 'E' quantified by measuring their distribution of nearest neighbor distances. An increase in HaCaT dispersion represents a reduction in their segregation from HT1080 cells. $\left(n=3\right.$, error bars $=S E M,{ }^{* *} P<$ $0.01,{ }^{*} \mathrm{P}<0.05$, Student's t-test).

(E) Long term culture of HaCaT cells (green) alone or during co-culture with NIH3T3 or HT1080 cells (unlabeled). Note the specific increase in cluster size of HaCaT cells cultured alone or in the presence of HT1080 cells. Scale bar $=100 \mu \mathrm{m}$. 
(F) Quantification of area occupied by HaCaT cells from the images in 'C'. Note that HaCaT cells are prevented from expanding specifically when co-cultured with NIH3T3 cells.

\section{Figure S1. Related to Figure 1}

592

593

594

(A) Screenshots from representative movies of a confrontation assay in which Human Corneal Epithelial Cells (HCE) are allowed to collide with fibrosarcoma cells (HT1080). HCE cells are labelled green and HT1080 cells are labelled magenta. Scale bar $=100 \mu \mathrm{m}$.

\section{Figure S2. Related to Figure 2}

(A) Western blot confirming successful knockdown of EphB2 protein in HT1080 cells $48 \mathrm{~h}$ post-treatment with siRNA compared with non-transfected cells (wild-type).

(B) Screenshots from representative movies of a confrontation assay in which epithelial cells (HaCaT) are allowed to collide with either control, EphB2 or ERK1/2 knockdown fibrosarcoma cells (HT1080). Epithelial cells (HaCaT) are labelled green and fibrosarcoma (HT1080) cells are labelled magenta. The red line indicates the position of initial cell-cell contact. Scale bars $=100 \mu \mathrm{m}$.

(C) Western blot confirming successful knockdown of ERK1/2 protein in HT1080 cells $48 \mathrm{~h}$ post-treatment with siRNA compared with non-transfected cells (wildtype).

(D) Quantification of non-colliding HT1080 cell speed in cells transfected with either EphB2 or ERK1/2 siRNA compared with non-transfected cells (wild-type). ( $n=5$ 10 cells, error bars $=$ SEM, ns $=$ not statistically significant, Student's t-test).

(E) Quantification of non-colliding HT1080 cell persistence in cells transfected with 
612 either EphB2 or ERK1/2 siRNA compared with non-transfected cells (wild-type).

$613(n=5-10$ cells, error bars $=$ SEM, $n s=$ not statistically significant, Student's $t-$

614 test).

615

616 Figure S3. Related to Figure 2

617 (A) Screenshots from representative movies of a confrontation assay in which 618 epithelial cells (HaCaT) are allowed to collide with fibrosarcoma cells (HT1080) 619 after blebbistatin treatment to inhibit Myosin II. HaCaT cells are labelled green and HT1080 cells are labelled magenta. Scale bar $=100 \mu \mathrm{m}$.

(B) Quantification of the average speed of the HT1080 cell population in the confrontation assay before the collision occurs. Cells treated with Blebbistatin migrated with a higher average pre-collision speed than untreated cells, indicating that blebbistatin has been effective in myosin II inhibition ( $n=3$, error bars $=$ SEM,$P^{*}<0.05$, Student's t-test $)$.

629

$630 \quad$ Figure S4. Related to Figure 4

631 (A) Directional vector-field plots for NIH3T3 and HT1080 cells (taken at time $15 \mathrm{~h}$ from the respective movies in Video S4).

(B) Quantification of the local alignment of cell velocities of NIH3T3 or HT1080 cells during co-culture with HaCaT cells. A positive correlation corresponds to polar alignment of velocities and a negative correlation an anti-parallel alignment. Note 

${ }^{* * *} \mathrm{P}<0.001$, Mann-Whitney test).

(C) Images of co-cultures of epithelial cells (HaCaT) with fibrosarcoma cells (HT1080) comparing DMSO control with U0126 treatment to inhibit ERK1/2 signaling.

(D) Quantification of HaCaT cell numbers at 6 and $24 \mathrm{~h}$ which indicates that U0126 inhibition of ERK does not selectively impact the growth rate of HaCaT cells. $(n=$ 3, error bars $=$ SEM, ns = not statistically significant, Student's t-test).

(E) The dispersion of HaCaT cells quantified by measuring their distribution of nearest neighbor distances. An increase in HaCaT dispersion represents a reduction in their segregation from HT1080 cells. $(n=3$, error bars $=$ SEM, * $P<$ 0.05 , Student's t-test).

(F) Basal proliferation rates of NIH3T3 and HT1080 cells measured over a 5-day period which demonstrates that rates do not differ between the two cell-types. ( $\mathrm{n}$

\section{Video S1. Related to Figure 1}

654 Example movies of the confrontation assay following barrier removal. HaCaT epithelial cells are labelled green and either NIH3T3 fibroblasts or HT1080 fibrosarcoma cells are labelled magenta. Time steps $=1 \mathrm{~h}$. Scale bar $=100 \mu \mathrm{m}$.

\section{Video S2. Related to Figure 1}

659 Particle Image Velocimetry (PIV) heat-map of the HaCaT and HT1080 interaction 660 in Video S1 showing an increase in speed of the entire population of HaCaT cells 

after colliding with HT1080 cells. Blue to red represents a shift from low to high instantaneous velocity.

663

664 Video S3. Related to Figure 2

665 Examples of DIC movies showing individual HT1080 fibrosarcoma cells (Control, 666 EphB2 or ERK1/2 knockdown) colliding with HaCaT epithelial cells or homotypic 667 collisions between HT1080 cells. Red lines represent manual tracking used for 668 the kinematics analysis presented in Figure 2. Time steps $=1 \mathrm{~min}$. Scale Bar = $66920 \mu \mathrm{m}$.

670

\section{Video S4. Related to Figure 4}

672 (Top) Example movies of the mixing assay. HaCaT epithelial cells are labelled in green, other cells magenta. Time steps $=10 \mathrm{~min}$. Scale bar $=100 \mu \mathrm{m}$.

674 (Bottom) Tracks of NIH3T3 fibroblasts (left) and HT1080 fibrosarcoma cells (right) 675 from the movies above. Blue through red coloration of tracks represents 676 temporal position in movie to show that, in the HT1080 case (bottom right), cells eventually become segregated from HaCaT cells (not labelled). 



$24 h$

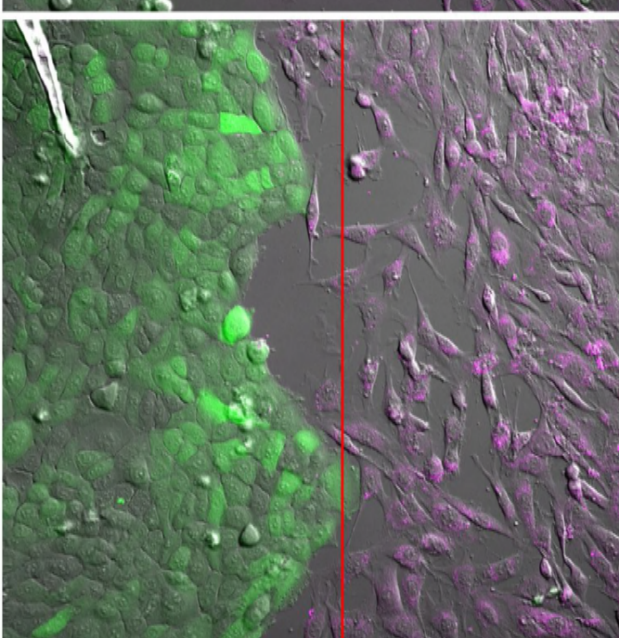

C
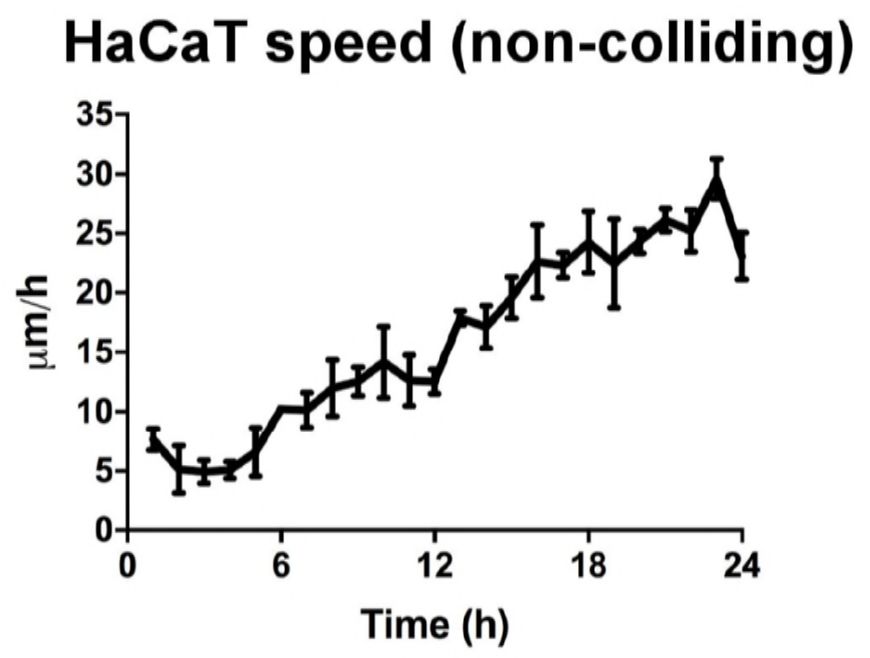

D

HaCaT speed (colliding)

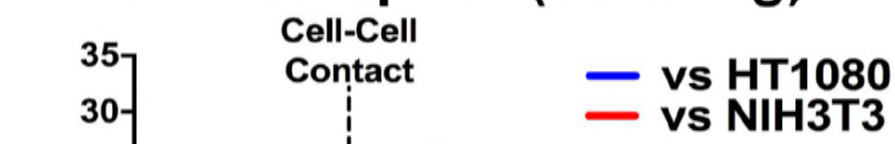

$36 h$


48h 
A $\begin{gathered}\text { Acceleration } \\ 0 \mathrm{~min}\end{gathered}$

HT1080 vs HaCaT

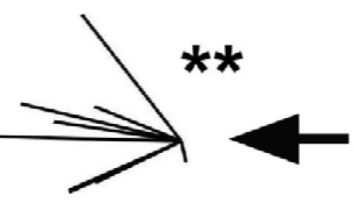

NIH3T3 vs $\mathrm{HaCaT}$

HT1080 vs HT1080

HT1080 (ERK1/2 KD) vs $\mathrm{HaCaT}$

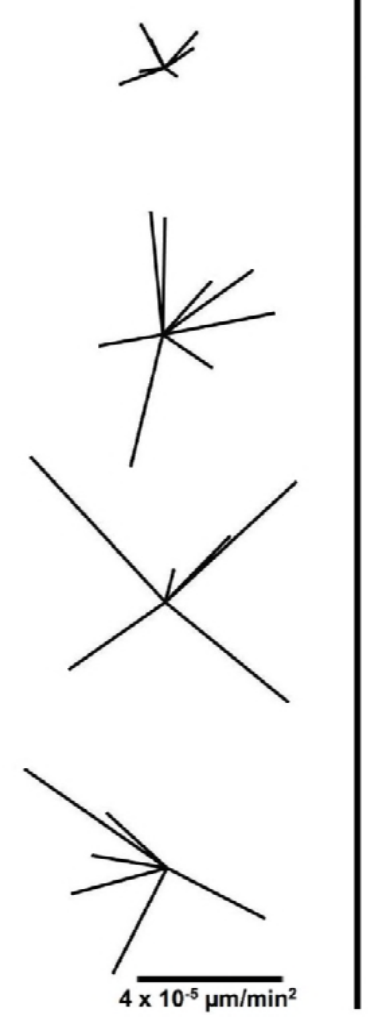

C



Individual cell collisions

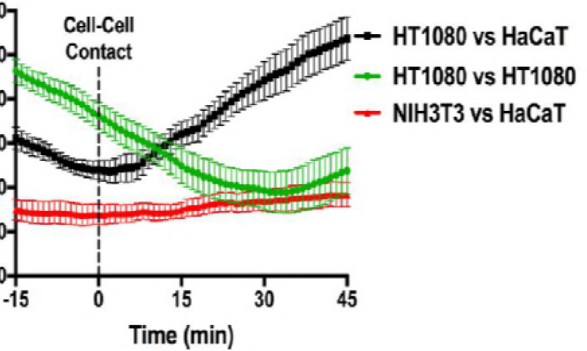

D Individual cell collisions

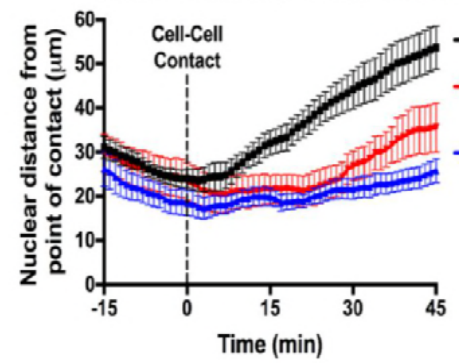

$+10 \mathrm{~min}$

本
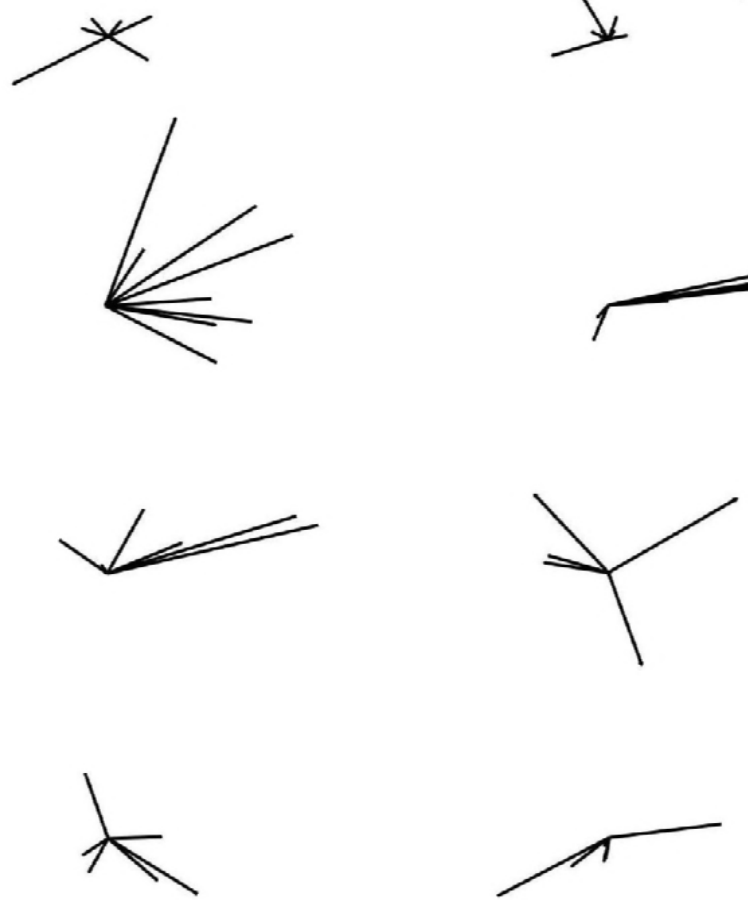

$2 \mu \mathrm{m} / \mathrm{min}$
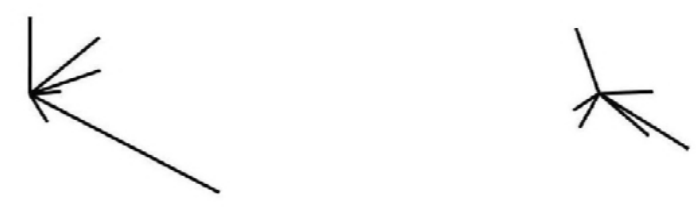

- HT1080 vs HaCaT

- HT1080 (EphB2. siRNA) vs $\mathrm{HaCaT}$

- HT1080 (ERK1/2. siRNA) vs HaCaT
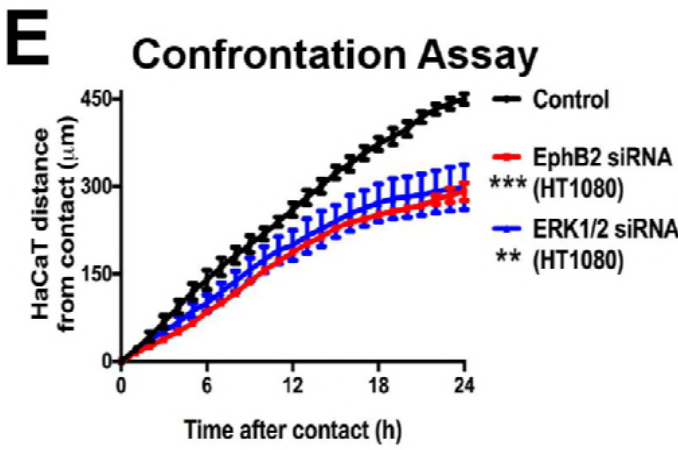
